\title{
PAC Statistical Model Checking for Markov Decision Processes and Stochastic Games
}

\author{
Pranav Ashok, Jan Křetínský, \\ and Maximilian Weininger ${ }^{(凶)}$ \\ Technical University of Munich, Munich, Germany \\ maxi.weininger@tum.de
}

\begin{abstract}
Statistical model checking (SMC) is a technique for analysis of probabilistic systems that may be (partially) unknown. We present an SMC algorithm for (unbounded) reachability yielding probably approximately correct (PAC) guarantees on the results. We consider both the setting (i) with no knowledge of the transition function (with the only quantity required a bound on the minimum transition probability) and (ii) with knowledge of the topology of the underlying graph. On the one hand, it is the first algorithm for stochastic games. On the other hand, it is the first practical algorithm even for Markov decision processes. Compared to previous approaches where PAC guarantees require running times longer than the age of universe even for systems with a handful of states, our algorithm often yields reasonably precise results within minutes, not requiring the knowledge of mixing time.
\end{abstract}

\section{Introduction}

Statistical model checking (SMC) [YS02a] is an analysis technique for probabilistic systems based on

1. simulating finitely many finitely long runs of the system,

2. statistical analysis of the obtained results,

3. yielding a confidence interval/probably approximately correct (PAC) result on the probability of satisfying a given property, i.e., there is a non-zero probability that the bounds are incorrect, but they are correct with probability that can be set arbitrarily close to 1 .

One of the advantages is that it can avoid the state-space explosion problem, albeit at the cost of weaker guarantees. Even more importantly, this technique is applicable even when the model is not known (black-box setting) or only

This research was funded in part by TUM IGSSE Grant 10.06 (PARSEC), the Czech Science Foundation grant No. 18-11193S, and the German Research Foundation (DFG) project KR 4890/2-1 "Statistical Unbounded Verification".

(C) The Author(s) 2019

I. Dillig and S. Tasiran (Eds.): CAV 2019, LNCS 11561, pp. 497-519, 2019.

https://doi.org/10.1007/978-3-030-25540-4_29 
qualitatively known (grey-box setting), where the exact transition probabilities are unknown such as in many cyber-physical systems.

In the basic setting of Markov chains [Nor98] with (time- or step-)bounded properties, the technique is very efficient and has been applied to numerous domains, e.g. biological [JCL+09,PGL+13], hybrid [ZPC10,DDL+12,EGF12, Lar12] or cyber-physical [BBB $+10, \mathrm{CZ11}, \mathrm{DDL}+13]$ systems and a substantial tool support is available [JLS12, BDL+12, BCLS13, BHH12]. In contrast, whenever either (i) infinite time-horizon properties, e.g. reachability, are considered or (ii) non-determinism is present in the system, providing any guarantees becomes significantly harder.

Firstly, for infinite time-horizon properties we need a stopping criterion such that the infinite-horizon property can be reliably evaluated based on a finite prefix of the run yielded by simulation. This can rely on the the complete knowledge of the system (white-box setting) [YCZ10,LP08], the topology of the system (grey box) [YCZ10,HJB+10], or a lower bound $p_{\min }$ on the minimum transition probability in the system (black box) [DHKP16, BCC+14].

Secondly, for Markov decision processes (MDP) [Put14] with (non-trivial) non-determinism, [HMZ+12] and [LP12] employ reinforcement learning [SB98] in the setting of bounded properties or discounted (and for the purposes of approximation thus also bounded) properties, respectively. The latter also yields PAC guarantees.

Finally, for MDP with unbounded properties, [BFHH11] deals with MDP with spurious non-determinism, where the way it is resolved does not affect the desired property. The general non-deterministic case is treated in [FT14, $\mathrm{BCC}+14]$, yielding PAC guarantees. However, the former requires the knowledge of mixing time, which is at least as hard to compute; the algorithm in the latter is purely theoretical since before a single value is updated in the learning process, one has to simulate longer than the age of universe even for a system as simple as a Markov chain with 12 states having at least 4 successors for some state.

Our contribution is an SMC algorithm with PAC guarantees for (i) MDP and unbounded properties, which runs for realistic benchmarks $[\mathrm{HKP}+19]$ and confidence intervals in orders of minutes, and (ii) is the first algorithm for stochastic games $(\mathrm{SG})$. It relies on different techniques from literature.

1. The increased practical performance rests on two pillars:

- extending early detection of bottom strongly connected components in Markov chains by [DHKP16] to end components for MDP and simple end components for SG;

- improving the underlying PAC Q-learning technique of $[S L W+06]$ :

(a) learning is now model-based with better information reuse instead of model-free, but in realistic settings with the same memory requirements,

(b) better guidance of learning due to interleaving with precise computation, which yields more precise value estimates.

(c) splitting confidence over all relevant transitions, allowing for variable width of confidence intervals on the learnt transition probabilities. 
2. The transition from algorithms for MDP to SG is possible via extending the over-approximating value iteration from $\mathrm{MDP}[\mathrm{BCC}+14]$ to $\mathrm{SG}$ by [KKKW18].

To summarize, we give an anytime PAC SMC algorithm for (unbounded) reachability. It is the first such algorithm for SG and the first practical one for MDP.

\section{Related Work}

Most of the previous efforts in SMC have focused on the analysis of properties with bounded horizon [YS02a, SVA04, YKNP06, JCL+09, JLS12, BDL+12].

SMC of unbounded properties was first considered in [HLMP04] and the first approach was proposed in [SVA05], but observed incorrect in [HJB+10]. Notably, in [YCZ10] two approaches are described. The first approach proposes to terminate sampled paths at every step with some probability $p_{\text {term }}$ and reweight the result accordingly. In order to guarantee the asymptotic convergence of this method, the second eigenvalue $\lambda$ of the chain and its mixing time must be computed, which is as hard as the verification problem itself and requires the complete knowledge of the system (white box setting). The correctness of [LP08] relies on the knowledge of the second eigenvalue $\lambda$, too. The second approach of [YCZ10] requires the knowledge of the chain's topology (grey box), which is used to transform the chain so that all potentially infinite paths are eliminated. In $[\mathrm{HJB}+10]$, a similar transformation is performed, again requiring knowledge of the topology. In [DHKP16], only (a lower bound on) the minimum transition probability $p_{\min }$ is assumed and PAC guarantees are derived. While unbounded properties cannot be analyzed without any information on the system, knowledge of $p_{\min }$ is a relatively light assumption in many realistic scenarios [DHKP16]. For instance, bounds on the rates for reaction kinetics in chemical reaction systems are typically known; for models in the PRISM language [KNP11], the bounds can be easily inferred without constructing the respective state space. In this paper, we thus adopt this assumption.

In the case with general non-determinism, one approach is to give the nondeterminism a probabilistic semantics, e.g., using a uniform distribution instead, as for timed automata in [DLL+11a,DLL+11b, Lar13]. Others [LP12, HMZ+12, $\mathrm{BCC}+14]$ aim to quantify over all strategies and produce an $\epsilon$-optimal strategy. In $[\mathrm{HMZ}+12]$, candidates for optimal strategies are generated and gradually improved, but "at any given point we cannot quantify how close to optimal the candidate scheduler is" (cited from $[\mathrm{HMZ}+12]$ ) and the algorithm "does not in general converge to the true optimum" (cited from [LST14]). Further, [LST14,DLST15,DHS18] randomly sample compact representation of strategies, resulting in useful lower bounds if $\varepsilon$-schedulers are frequent. [HPS+19] gives a convergent model-free algorithm (with no bounds on the current error) and identifies that the previous [SKC+14] "has two faults, the second of which also affects approaches [...] [HAK18, HAK19]".

Several approaches provide SMC for MDPs and unbounded properties with PAC guarantees. Firstly, similarly to [LP08, YCZ10], [FT14] requires (1) the 
mixing time $T$ of the MDP. The algorithm then yields PAC bounds in time polynomial in $T$ (which in turn can of course be exponential in the size of the MDP). Moreover, the algorithm requires (2) the ability to restart simulations also in non-initial states, (3) it only returns the strategy once all states have been visited (sufficiently many times), and thus (4) requires the size of the state space $|S|$. Secondly, [BCC+14], based on delayed Q-learning (DQL) [SLW+06], lifts the assumptions (2) and (3) and instead of (1) mixing time requires only (a bound on) the minimum transition probability $p_{\min }$. Our approach additionally lifts the assumption (4) and allows for running times faster than those given by $T$, even without the knowledge of $T$.

Reinforcement learning (without PAC bounds) for stochastic games has been considered already in [LN81,Lit94,BT99]. [WT16] combines the special case of almost-sure satisfaction of a specification with optimizing quantitative objectives. We use techniques of [KKKW18], which however assumes access to the transition probabilities.

\section{Preliminaries}

\subsection{Stochastic Games}

A probability distribution on a finite set $X$ is a mapping $\delta: X \rightarrow[0,1]$, such that $\sum_{x \in X} \delta(x)=1$. The set of all probability distributions on $X$ is denoted by $\mathcal{D}(X)$. Now we define turn-based two-player stochastic games. As opposed to the notation of e.g. [Con92], we do not have special stochastic nodes, but rather a probabilistic transition function.

Definition 1 (SG). $A$ stochastic game $(\mathrm{SG})$ is a tuple $\mathrm{G}=\left(\mathrm{S}, \mathrm{S}_{\square}, \mathrm{S}_{\bigcirc}, \mathrm{S}_{0}, \mathrm{~A}, \mathrm{Av}, \mathbb{T}\right)$, where $\mathrm{S}$ is a finite set of states partitioned ${ }^{1}$ into the sets $\mathrm{S}_{\square}$ and $\mathrm{S}_{\bigcirc}$ of states of the player Maximizer and Minimizer ${ }^{2}$, respectively $\mathrm{s}_{0} \in \mathrm{S}$ is the initial state, $\mathrm{A}$ is a finite set of actions, $\mathrm{Av}: \mathrm{S} \rightarrow 2^{\mathrm{A}}$ assigns to every state a set of available actions, and $\mathbb{T}: S \times A \rightarrow \mathcal{D}(S)$ is a transition function that given a state $s$ and an action $a \in A v(s)$ yields a probability distribution over successor states. Note that for ease of notation we write $\mathbb{T}(s, a, t)$ instead of $\mathbb{T}(\mathrm{s}, \mathrm{a})(\mathrm{t})$.

A Markov decision process (MDP) is a special case of $\mathrm{SG}$ where $\mathrm{S}_{\bigcirc}=\emptyset$. A Markov chain (MC) can be seen as a special case of an MDP, where for all $\mathrm{s} \in \mathrm{S}:|\operatorname{Av}(\mathrm{s})|=1$. We assume that $\mathrm{SG}$ are non-blocking, so for all states $\mathrm{s}$ we have $\operatorname{Av}(s) \neq \emptyset$.

For a state $s$ and an available action $a \in A v(s)$, we denote the set of successors by $\operatorname{Post}(\mathrm{s}, \mathrm{a}):=\{\mathrm{t} \mid \mathbb{T}(\mathrm{s}, \mathrm{a}, \mathrm{t})>0\}$. We say a state-action pair (s, a) is an exit of a set of states $T$, written (s, a) exits $T$, if $\exists \mathrm{t} \in \operatorname{Post}(\mathrm{s}, \mathrm{a}): \mathrm{t} \notin T$, i.e., if with some probability a successor outside of $T$ could be chosen.

We consider algorithms that have a limited information about the SG.

${ }^{1}$ I.e., $\mathrm{S}_{\square} \subseteq \mathrm{S}, \mathrm{S}_{\bigcirc} \subseteq \mathrm{S}, \mathrm{S}_{\square} \cup \mathrm{S}_{\bigcirc}=\mathrm{S}$, and $\mathrm{S}_{\square} \cap \mathrm{S}_{\bigcirc}=\emptyset$.

2 The names are chosen, because Maximizer maximizes the probability of reaching a given target state, and Minimizer minimizes it. 
Definition 2 (Black box and grey box). An algorithm inputs an $S G$ as black box if it cannot access the whole tuple, but

- it knows the initial state,

- for a given state, an oracle returns its player and available action,

- given a state $\mathrm{s}$ and action $\mathrm{a}$, it can sample a successor $\mathrm{t}$ according to $\mathbb{T}(\mathrm{s}, \mathrm{a}),^{3}$

- it knows $p_{\min } \leq \min _{\mathrm{s} \in \mathrm{S}, \mathrm{a} \in \mathrm{Av}(\mathrm{s})} \mathbb{T}(\mathrm{s}, \mathrm{a}, \mathrm{t})$, an under-approximation of the minimum transition probability.

When input as grey box it additionally knows the number $|\operatorname{Post}(\mathrm{s}, \mathrm{a})|$ of successors for each state $\mathrm{s}$ and action $\mathrm{a}^{4}$

The semantics of SG is given in the usual way by means of strategies and the induced Markov chain [BK08] and its respective probability space, as follows. An infinite path $\rho$ is an infinite sequence $\rho=\mathrm{s}_{0} \mathrm{a}_{0} \mathrm{~s}_{1} \mathrm{a}_{1} \cdots \in(\mathrm{S} \times \mathrm{A})^{\omega}$, such that for every $i \in \mathbb{N}, \mathrm{a}_{i} \in \operatorname{Av}\left(\mathrm{s}_{i}\right)$ and $\mathrm{s}_{i+1} \in \operatorname{Post}\left(\mathrm{s}_{i}, \mathrm{a}_{i}\right)$.

A strategy of Maximizer or Minimizer is a function $\sigma: \mathrm{S}_{\square} \rightarrow \mathcal{D}(\mathrm{A})$ or $\mathrm{S}_{\bigcirc} \rightarrow$ $\mathcal{D}(\mathrm{A})$, respectively, such that $\sigma(\mathrm{s}) \in \mathcal{D}(\operatorname{Av}(\mathrm{s}))$ for all s. Note that we restrict to memoryless/positional strategies, as they suffice for reachability in SGs [CH12].

A pair $(\sigma, \tau)$ of strategies of Maximizer and Minimizer induces a Markov chain $\mathrm{G}^{\sigma, \tau}$ with states $\mathrm{S}, \mathrm{s}_{0}$ being initial, and the transition function $\mathbb{T}(\mathrm{s})(\mathrm{t})=$ $\sum_{\mathrm{a} \in \operatorname{Av}(\mathrm{s})} \sigma(\mathrm{s})(\mathrm{a}) \cdot \mathbb{T}(\mathrm{s}, \mathrm{a}, \mathrm{t})$ for states of Maximizer and analogously for states of Minimizer, with $\sigma$ replaced by $\tau$. The Markov chain induces a unique probability distribution $\mathbb{P}^{\sigma, \tau}$ over measurable sets of infinite paths [BK08, Ch. 10].

\subsection{Reachability Objective}

For a goal set Goal $\subseteq \mathrm{S}$, we write $\diamond$ Goal $:=\left\{\mathrm{s}_{0} \mathrm{a}_{0} \mathrm{~s}_{1} \mathrm{a}_{1} \cdots \mid \exists i \in \mathbb{N}: \mathrm{s}_{i} \in\right.$ Goal $\}$ to denote the (measurable) set of all infinite paths which eventually reach Goal. For each $\mathrm{s} \in \mathrm{S}$, we define the value in $\mathrm{s}$ as

$$
\mathrm{V}(\mathrm{s}):=\sup _{\sigma} \inf _{\tau} \mathbb{P}_{s}^{\sigma, \tau}(\diamond \mathrm{Goal})=\inf _{\tau} \sup _{\sigma} \mathbb{P}_{s}^{\sigma, \tau}(\diamond \mathrm{Goal})
$$

where the equality follows from [Mar75]. We are interested in $\mathrm{V}\left(\mathrm{s}_{0}\right)$, its $\varepsilon$-approximation and the corresponding $(\varepsilon$-)optimal strategies for both players.

${ }^{3}$ Up to this point, this definition conforms to black box systems in the sense of [SVA04] with sampling from the initial state, being slightly stricter than [YS02a] or [RP09], where simulations can be run from any desired state. Further, we assume that we can choose actions for the adversarial player or that she plays fairly. Otherwise the adversary could avoid playing her best strategy during the SMC, not giving SMC enough information about her possible behaviours.

4 This requirement is slightly weaker than the knowledge of the whole topology, i.e. Post $(\mathrm{s}, \mathrm{a})$ for each $\mathrm{s}$ and $\mathrm{a}$. 
Let Zero be the set of states, from which there is no finite path to any state in Goal. The value function $V$ satisfies the following system of equations, which is referred to as the Bellman equations:

$$
V(s)= \begin{cases}\max _{a \in A v(s)} V(s, a) & \text { if } s \in S_{\square} \\ \min _{a \in A v(s)} V(s, a) & \text { if } s \in S_{\bigcirc} \\ 1 & \text { if } s \in \text { Goal } \\ 0 & \text { if } s \in \text { Zero }\end{cases}
$$

with the abbreviation $\mathrm{V}(\mathrm{s}, \mathrm{a}):=\sum_{s^{\prime} \in S} \mathbb{T}\left(\mathrm{s}, \mathrm{a}, \mathrm{s}^{\prime}\right) \cdot \mathrm{V}\left(\mathrm{s}^{\prime}\right)$. Moreover, $\mathrm{V}$ is the least solution to the Bellman equations, see e.g. [CH08].

\subsection{Bounded and Asynchronous Value Iteration}

The well known technique of value iteration, e.g. [Put14, RF91], works by starting from an under-approximation of value function and then applying the Bellman equations. This converges towards the least fixpoint of the Bellman equations, i.e. the value function. Since it is difficult to give a convergence criterion, the approach of bounded value iteration (BVI, also called interval iteration) was developed for MDP [BCC+14,HM17] and SG [KKKW18]. Beside the underapproximation, it also updates an over-approximation according to the Bellman equations. The most conservative over-approximation is to use an upper bound of 1 for every state. For the under-approximation, we can set the lower bound of target states to 1 ; all other states have a lower bound of 0 . We use the function INITIALIZE_BOUNDS in our algorithms to denote that the lower and upper bounds are set as just described; see [AKW19, Algorithm 8] for the pseudocode. Additionally, BVI ensures that the over-approximation converges to the least fixpoint by taking special care of end components, which are the reason for not converging to the true value from above.

Definition 3 (End component (EC)). A non-empty set $T \subseteq \mathrm{S}$ of states is an end component (EC) if there is a non-empty set $B \subseteq \bigcup_{\mathrm{s} \in T} \mathrm{Av}(s)$ of actions such that (i) for each $\mathrm{s} \in T, \mathrm{a} \in B \cap \mathrm{Av}(\mathrm{s})$ we do not have (s, a) exits $T$ and (ii) for each $\mathrm{s}, \mathrm{s}^{\prime} \in T$ there is a finite path $\mathrm{w}=\mathrm{sa}_{0} \ldots \mathrm{a}_{n} \mathrm{~s}^{\prime} \in(T \times B)^{*} \times T$, i.e. the path stays inside $T$ and only uses actions in $B$.

Intuitively, ECs correspond to bottom strongly connected components of the Markov chains induced by possible strategies, so for some pair of strategies all possible paths starting in the EC remain there. An end component $T$ is a maximal end component $(M E C)$ if there is no other end component $T^{\prime}$ such that $T \subseteq T^{\prime}$. Given an SG G, the set of its MECs is denoted by $\operatorname{MEC}(\mathrm{G})$.

Note that, to stay in an EC in an SG, the two players would have to cooperate, since it depends on the pair of strategies. To take into account the adversarial behaviour of the players, it is also relevant to look at a subclass of ECs, the so called simple end components, introduced in [KKKW18]. 
Definition 4 (Simple end component (SEC) [KKKW18]). An EC T is called simple, if for all $\mathrm{s} \in T$ it holds that $\mathrm{V}(\mathrm{s})=$ bestExit $(T, \mathrm{~V})$, where

$$
\text { bestExit }(T, f):= \begin{cases}1 & \text { if } T \cap \text { Goal } \neq \emptyset \\ \max _{\substack{\mathrm{s} \in T \cap \mathrm{S}_{\square} \\(\mathrm{s}, \mathrm{a}) \operatorname{exits} T}} f(\mathrm{~s}, \mathrm{a}) & \text { else }\end{cases}
$$

is called the best exit (of Maximizer) from $T$ according to the function $f: S \rightarrow \mathbb{R}$. To handle the case that there is no exit of Maximizer in $T$ we set $\max _{\emptyset}=0$.

Intuitively, SECs are ECs where Minimizer does not want to use any of her exits, as all of them have a greater value than the best exit of Maximizer. Assigning any value between those of the best exits of Maximizer and Minimizer to all states in the EC is a solution to the Bellman equations, because both players prefer remaining and getting that value to using their exits [KKKW18, Lemma 1]. However, this is suboptimal for Maximizer, as the goal is not reached if the game remains in the EC forever. Hence we "deflate" the upper bounds of SECs, i.e. reduce them to depend on the best exit of Maximizer. $T$ is called maximal simple end component (MSEC), if there is no SEC $T^{\prime}$ such that $T \subsetneq T^{\prime}$. Note that in MDPs, treating all MSECs amounts to treating all MECs.

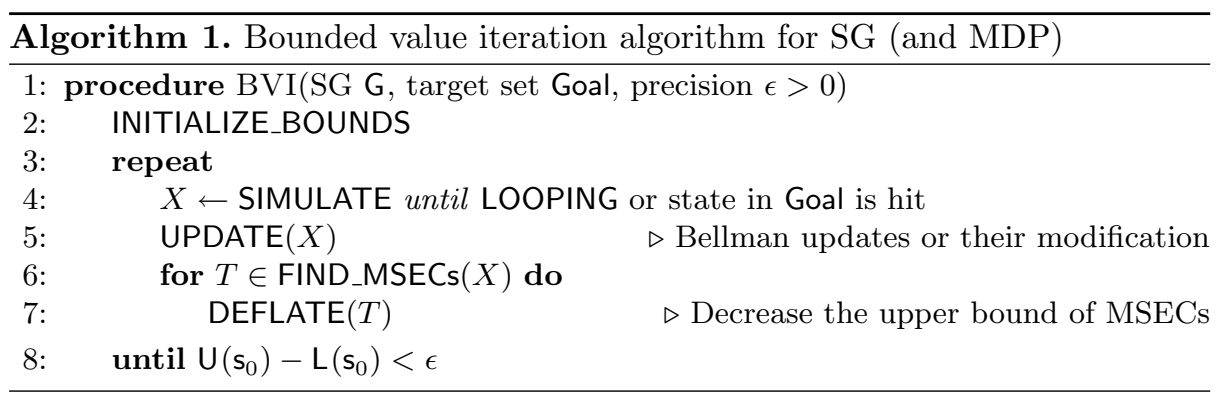

Algorithm 1 rephrases that of [KKKW18] and describes the general structure of all bounded value iteration algorithms that are relevant for this paper. We discuss it here since all our improvements refer to functions (in capitalized font) in it. In the next section, we design new functions, pinpointing the difference to the other papers. The pseudocode of the functions adapted from the other papers can be found, for the reader's convenience, in [AKW19, Appendix A]. Note that to improve readability, we omit the parameters G, Goal, L and $U$ of the functions in the algorithm.

Bounded Value Iteration: For the standard bounded value iteration algorithm, Line 4 does not run a simulation, but just assigns the whole state space $S$ to $X^{5}$. Then it updates all values according to the Bellman equations.

\footnotetext{
${ }^{5}$ Since we mainly talk about simulation based algorithms, we included this line to make their structure clearer.
} 
After that it finds all the problematic components, the MSECs, and "deflates" them as described in [KKKW18], i.e. it reduces their values to ensure the convergence to the least fixpoint. This suffices for the bounds to converge and the algorithm to terminate [KKKW18, Theorem 2].

Asynchronous Bounded Value Iteration: To tackle the state space explosion problem, asynchronous simulation/learning-based algorithms have been developed [MLG05, BCC+14,KKKW18]. The idea is not to update and deflate all states at once, since there might be too many, or since we only have limited information. Instead of considering the whole state space, a path through the SG is sampled by picking in every state one of the actions that look optimal according to the current over-/under-approximation and then sampling a successor of that action. This is repeated until either a target is found, or until the simulation is looping in an EC; the latter case occurs if the heuristic that picks the actions generates a pair of strategies under which both players only pick staying actions in an EC. After the simulation, only the bounds of the states on the path are updated and deflated. Since we pick actions which look optimal in the simulation, we almost surely find an $\epsilon$-optimal strategy and the algorithm terminates $[\mathrm{BCC}+14$, Theorem 3].

\section{Algorithm}

\subsection{Model-Based}

Given only limited information, updating cannot be done using $\mathbb{T}$, since the true probabilities are not known. The approach of $[\mathrm{BCC}+14]$ is to sample for a high number of steps and accumulate the observed lower and upper bounds on the true value function for each state-action pair. When the number of samples is large enough, the average of the accumulator is used as the new estimate for the state-action pair, and thus the approximations can be improved and the results back-propagated, while giving statistical guarantees that each update was correct. However, this approach has several drawbacks, the biggest of which is that the number of steps before an update can occur is infeasibly large, often larger than the age of the universe, see Table 1 in Sect. 4.

Our improvements to make the algorithm practically usable are linked to constructing a partial model of the given system. That way, we have more information available on which we can base our estimates, and we can be less conservative when giving bounds on the possible errors. The shift from model-free to model-based learning asymptotically increases the memory requirements from $\mathcal{O}(|\mathrm{S}| \cdot|\mathrm{A}|)$ (as in $[\mathrm{SLW}+06, \mathrm{BCC}+14])$ to $\mathcal{O}\left(|\mathrm{S}|^{2} \cdot|\mathrm{A}|\right)$. However, for systems where each action has a small constant bound on the number of successors, which is typical for many practical systems, e.g. classical PRISM benchmarks, it is still $\mathcal{O}(|\mathrm{S}| \cdot|\mathrm{A}|)$ with a negligible constant difference.

We thus track the number of times some successor $t$ has been observed when playing action a from state $s$ in a variable $\#(s, a, t)$. This implicitly induces the number of times each state-action pair $(\mathrm{s}, \mathrm{a})$ has been played $\#(\mathrm{~s}, \mathrm{a})=$ 
$\sum_{t \in S} \#(s, a, t)$. Given these numbers we can then calculate probability estimates for every transition as described in the next subsection. They also induce the set of all states visited so far, allowing us to construct a partial model of the game. See [AKW19, Appendix A.2] for the pseudo-code of how to count the occurrences during the simulations.

\subsection{Safe Updates with Confidence Intervals Using Distributed Error Probability}

We use the counters to compute a lower estimate of the transition probability for some error tolerance $\delta_{\mathbb{T}}$ as follows: We view sampling t from state-action pair $(\mathrm{s}, \mathrm{a})$ as a Bernoulli sequence, with success probability $\mathbb{T}(\mathrm{s}, \mathrm{a}, \mathrm{t})$, the number of trials \# $(\mathrm{s}, \mathrm{a})$ and the number of successes \# $(\mathrm{s}, \mathrm{a}, \mathrm{t})$. The tightest lower estimate we can give using the Hoeffding bound (see [AKW19, Appendix D.1]) is

$$
\widehat{\mathbb{T}}(\mathrm{s}, \mathrm{a}, \mathrm{t}):=\max \left(0, \frac{\#(s, a, t)}{\#(s, a)}-c\right),
$$

where the confidence width $c:=\sqrt{\frac{\ln \left(\delta_{\mathbb{T}}\right)}{-2 \#(s, a)}}$. Since $c$ could be greater than 1 , we limit the lower estimate to be at least 0 . Now we can give modified update equations:

$$
\begin{aligned}
& \widehat{\mathrm{L}}(\mathrm{s}, \mathrm{a}):=\sum_{\mathrm{t}: \#(\mathrm{~s}, \mathrm{a}, \mathrm{t})>0} \widehat{\mathbb{T}}(\mathrm{s}, \mathrm{a}, \mathrm{t}) \cdot \mathrm{L}(\mathrm{t}) \\
& \widehat{\mathrm{U}}(\mathrm{s}, \mathrm{a}):=\left(\sum_{\mathrm{t}: \#(\mathrm{~s}, \mathrm{a}, \mathrm{t})>0} \widehat{\mathbb{T}}(\mathrm{s}, \mathrm{a}, \mathrm{t}) \cdot \mathrm{U}(\mathrm{t})\right)+\left(1-\sum_{\mathrm{t}: \#(\mathrm{~s}, \mathrm{a}, \mathrm{t})>0} \widehat{\mathbb{T}}(\mathrm{s}, \mathrm{a}, \mathrm{t})\right)
\end{aligned}
$$

The idea is the same for both upper and lower bound: In contrast to the usual Bellman equation (see Sect. 2.2) we use $\widehat{\mathbb{T}}$ instead of $\mathbb{T}$. But since the sum of all the lower estimates does not add up to one, there is some remaining probability for which we need to under-/over-approximate the value it can achieve. We use

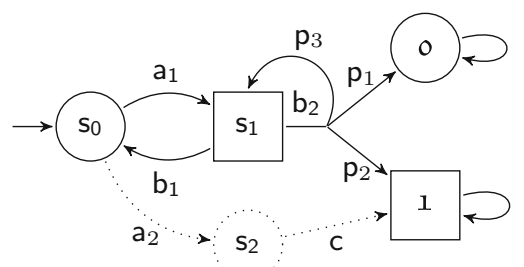

Fig. 1. A running example of an SG. The dashed part is only relevant for the later examples. For actions with only one successor, we do not depict the transition probability 1 (e.g. $\left.\mathbb{T}\left(\mathrm{s}_{0}, \mathrm{a}_{1}, \mathrm{~s}_{1}\right)\right)$. For state-action pair $\left(\mathrm{s}_{1}, \mathrm{~b}_{2}\right)$, the transition probabilities are parameterized and instantiated in the examples where they are used. 
the safe approximations 0 and 1 for the lower and upper bound respectively; this is why in $\hat{L}$ there is no second term and in $\widehat{U}$ the whole remaining probability is added. Algorithm 2 shows the modified update that uses the lower estimates; the proof of its correctness is in [AKW19, Appendix D.2].

Lemma 1 (UPDATE is correct). Given correct under- and over-approximations $\mathrm{L}, \mathrm{U}$ of the value function $\mathrm{V}$, and correct lower probability estimates $\widehat{\mathbb{T}}$, the under- and over-approximations after an application of UPDATE are also correct.

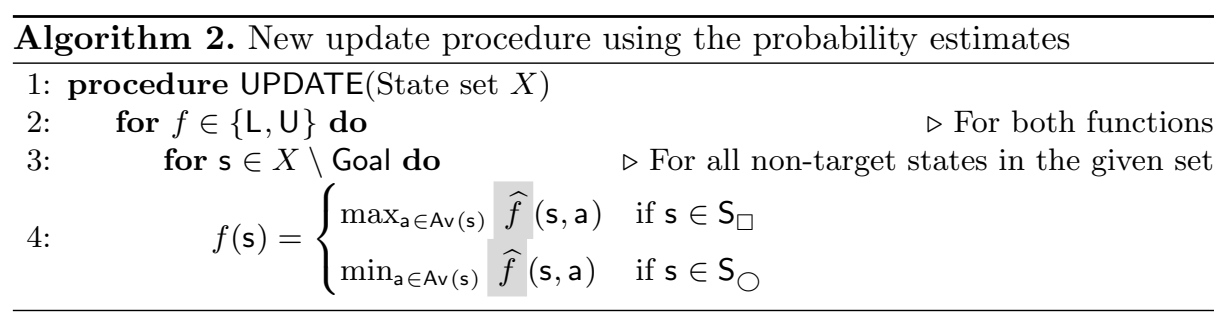

Example 1. We illustrate how the calculation works and its huge advantage over the approach from $[\mathrm{BCC}+14]$ on the $\mathrm{SG}$ from Fig. 1. For this example, ignore the dashed part and let $\mathrm{p}_{1}=\mathrm{p}_{2}=0.5$, i.e. we have no self loop, and an even chance to go to the target 1 or a sink 0 . Observe that hence $\mathrm{V}\left(\mathrm{s}_{0}\right)=\mathrm{V}\left(\mathrm{s}_{1}\right)=0.5$.

Given an error tolerance of $\delta=0.1$, the algorithm of [BCC+14] would have to sample for more than $10^{9}$ steps before it could attempt a single update. In contrast, assume we have seen 5 samples of action $b_{2}$, where 1 of them went to 1 and 4 of them to 0 . Note that, in a sense, we were unlucky here, as the observed averages are very different from the actual distribution. The confidence width for $\delta_{\mathbb{T}}=0.1$ and 5 samples is $\sqrt{\ln (0.1) /-2 \cdot 5} \approx 0.48$. So given that data, we get $\widehat{\mathbb{T}}\left(\mathrm{s}_{1}, \mathrm{~b}_{2}, \mathbf{l}\right)=\max (0,0.2-0.48)=0$ and $\widehat{\mathbb{T}}\left(\mathrm{s}_{1}, \mathrm{~b}_{2}, \mathrm{o}\right)=\max (0,0.8-0.48)=0.32$. Note that both probabilities are in fact lower estimates for their true counterpart.

Assume we already found out that $o$ is a sink with value 0 ; how we gain this knowledge is explained in the following subsections. Then, after getting only these 5 samples, UPDATE already decreases the upper bound of $\left(\mathrm{s}_{1}, \mathrm{~b}_{2}\right)$ to 0.68 , as we know that at least 0.32 of $\mathbb{T}\left(s_{1}, b_{2}\right)$ goes to the sink.

Given 500 samples of action $b_{2}$, the confidence width of the probability estimates already has decreased below 0.05. Then, since we have this confidence width for both the upper and the lower bound, we can decrease the total precision for $\left(s_{1}, b_{2}\right)$ to 0.1 , i.e. return an interval in the order of $[0.45 ; 0.55]$.

Summing up: with the model-based approach we can already start updating after very few steps and get a reasonable level of confidence with a realistic number of samples. In contrast, the state-of-the-art approach of $[\mathrm{BCC}+14]$ needs a very large number of samples even for this toy example.

Since for UPDATE we need an error tolerance for every transition, we need to distribute the given total error tolerance $\delta$ over all transitions in the current 
partial model. For all states in the explored partial model $\widehat{S}$ we know the number of available actions and can over-approximate the number of successors as $\frac{1}{p_{\min }}$. Thus the error tolerance for each transition can be set to $\delta_{\mathbb{T}}:=\frac{\delta \cdot p_{\min }}{|\{\mathrm{a} \mid \mathrm{s} \in \widehat{\mathrm{S}} \wedge \mathrm{a} \in \operatorname{Av}(\mathrm{s})\}|}$. This is illustrated in Example 4 in [AKW19, Appendix B].

Note that the fact that the error tolerance $\delta_{\mathbb{T}}$ for every transition is the same does not imply that the confidence width for every transition is the same, as the latter becomes smaller with increasing number of samples \#(s,a).

\subsection{Improved EC Detection}

As mentioned in the description of Algorithm 1, we must detect when the simulation is stuck in a bottom EC and looping forever. However, we may also stop simulations that are looping in some EC but still have a possibility to leave it; for a discussion of different heuristics from [BCC+14, KKKW18], see [AKW19, Appendix A.3].

We choose to define LOOPING as follows: Given a candidate for a bottom EC, we continue sampling until we are $\delta_{\mathbb{T}}$-sure (i.e. the error probability is smaller than $\delta_{\mathbb{T}}$ ) that we cannot leave it. Then we can safely deflate the EC, i.e. decrease all upper bounds to zero.

To detect that something is a $\delta_{\mathbb{T}}$-sure EC, we do not sample for the astronomical number of steps as in $[\mathrm{BCC}+14]$, but rather extend the approach to detect bottom strongly connected components from [DHKP16]. If in the EC-candidate $T$ there was some state-action pair (s, a) that actually has a probability to exit the $T$, that probability is at least $p_{\min }$. So after sampling $(\mathrm{s}, \mathrm{a})$ for $n$ times, the probability to overlook such a leaving transition is $\left(1-p_{\min }\right)^{n}$ and it should be smaller than $\delta_{\mathbb{T}}$. Solving the inequation for the required number of samples $n$ yields $n \geq \frac{\ln \left(\delta_{\mathbb{T}}\right)}{\ln \left(1-p_{\text {min }}\right)}$.

Algorithm 3 checks that we have seen all staying state-action pairs $n$ times, and hence that we are $\delta_{\mathbb{T}}$-sure that $T$ is an EC. Note that we restrict to staying state-action pairs, since the requirement for an EC is only that there exist staying actions, not that all actions stay. We further speed up the EC-detection, because we do not wait for $n$ samples in every simulation, but we use the aggregated counters that are kept over all simulations.

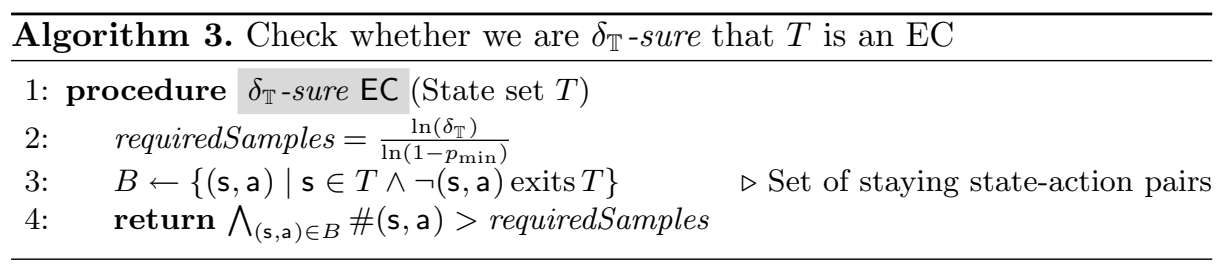

We stop a simulation, if LOOPING returns true, i.e. under the following three conditions: (i) We have seen the current state before in this simulation $(\mathrm{s} \in X)$, 
i.e. there is a cycle. (ii) This cycle is explainable by an EC $T$ in our current partial model. (iii) We are $\delta_{\mathbb{T}}$-sure that $T$ is an EC.

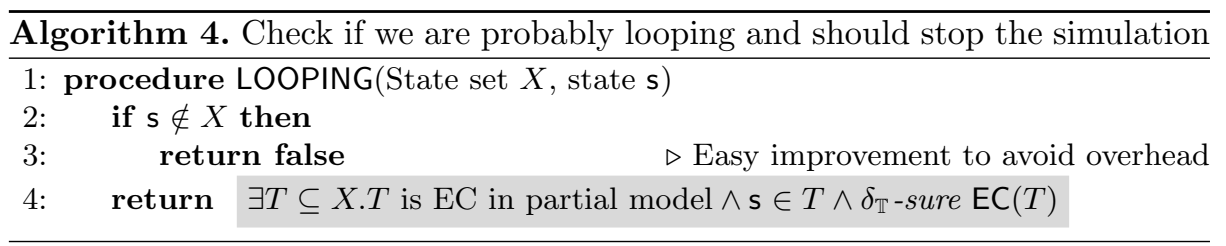

Example 2. For this example, we again use the SG from Fig. 1 without the dashed part, but this time with $\mathrm{p}_{1}=\mathrm{p}_{2}=\mathrm{p}_{3}=\frac{1}{3}$. Assume the path we simulated is $\left(s_{0}, a_{1}, s_{1}, b_{2}, s_{1}\right)$, i.e. we sampled the self-loop of action $b_{2}$. Then $\left\{s_{1}\right\}$ is a candidate for an EC, because given our current observation it seems possible that we will continue looping there forever. However, we do not stop the simulation here, because we are not yet $\delta_{\mathbb{T}}$-sure about this. Given $\delta_{\mathbb{T}}=0.1$, the required samples for that are 6 , since $\frac{\ln (0.1)}{\ln \left(1-\frac{1}{3}\right)}=5.6$. With high probability (greater than $\left.\left(1-\delta_{\mathbb{T}}\right)=0.9\right)$, within these 6 steps we will sample one of the other successors of $\left(s_{1}, b_{2}\right)$ and thus realise that we should not stop the simulation in $s_{1}$. If, on the other hand, we are in state $\mathcal{o}$ or if in state $s_{1}$ the guiding heuristic only picks $b_{1}$, then we are in fact looping for more than 6 steps, and hence we stop the simulation.

\subsection{Adapting to Games: Deflating MSECs}

To extend the algorithm of [BCC+14] to SGs, instead of collapsing problematic ECs we deflate them as in [KKKW18], i.e. given an MSEC, we reduce the upper bound of all states in it to the upper bound of the bestExit of Maximizer. In contrast to [KKKW18], we cannot use the upper bound of the bestExit based on the true probability, but only based on our estimates. Algorithm 5 shows how to deflate an MSEC and highlights the difference, namely that we use $\widehat{U}$ instead of $\mathrm{U}$.

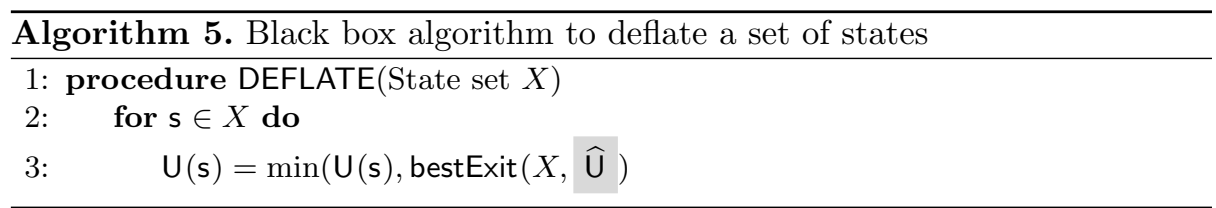

The remaining question is how to find MSECs. The approach of [KKKW18] is to find MSECs by removing the suboptimal actions of Minimizer according to the current lower bound. Since it converges to the true value function, all 
MSECs are eventually found [KKKW18, Lemma 2]. Since Algorithm 6 can only access the SG as a black box, there are two differences: We can only compare our estimates of the lower bound $\widehat{L}(s, a)$ to find out which actions are suboptimal. Additionally there is the problem that we might overlook an exit from an EC, and hence deflate to some value that is too small; thus we need to check that any state set FIND_MSECs returns is a $\delta_{\mathbb{T}}$-sure EC. This is illustrated in Example 3. For a bigger example of how all our functions work together, see Example 5 in [AKW19, Appendix B].

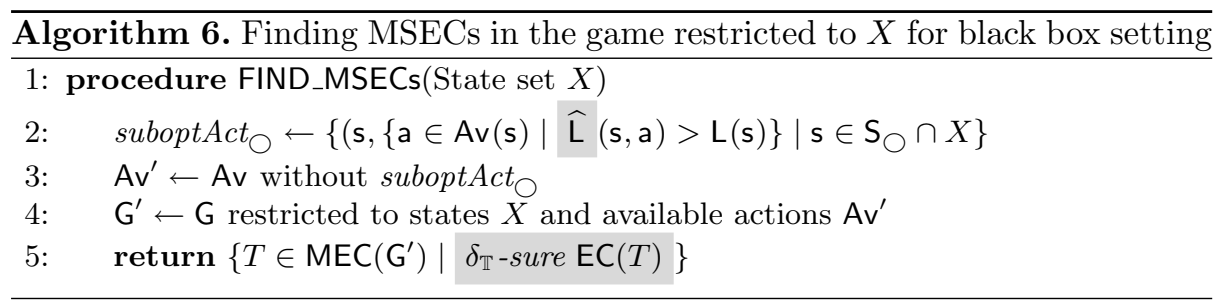

Example 3. For this example, we use the full SG from Fig. 1, including the dashed part, with $\mathrm{p}_{1}, \mathrm{p}_{2}>0$. Let $\left(\mathrm{s}_{0}, \mathrm{a}_{1}, \mathrm{~s}_{1}, \mathrm{~b}_{2}, \mathrm{~s}_{2}, \mathrm{~b}_{1}, \mathrm{~s}_{1}, \mathrm{a}_{2}, \mathrm{~s}_{2}, \mathrm{c}, \mathrm{l}\right)$ be the path generated by our simulation. Then in our partial view of the model, it seems as if $T=\left\{\mathrm{s}_{0}, \mathrm{~s}_{1}\right\}$ is an MSEC, since using $\mathrm{a}_{2}$ is suboptimal for the minimizing state $\mathrm{s}_{0}{ }^{6}$ and according to our current knowledge $\mathrm{a}_{1}, \mathrm{~b}_{1}$ and $\mathrm{b}_{2}$ all stay inside $T$. If we deflated $T$ now, all states would get an upper bound of 0 , which would be incorrect.

Thus in Algorithm 6 we need to require that $T$ is an $\mathrm{EC} \delta_{\mathbb{T}}$-surely. This was not satisfied in the example, as the state-action pairs have not been observed the required number of times. Thus we do not deflate $T$, and our upper bounds stay correct. Having seen $\left(s_{1}, b_{2}\right)$ the required number of times, we probably know that it is exiting $T$ and hence will not make the mistake.

\subsection{Guidance and Statistical Guarantee}

It is difficult to give statistical guarantees for the algorithm we have developed so far (i.e. Algorithm 1 calling the new functions from Sects. 3.2, 3.3 and 3.4). Although we can bound the error of each function, applying them repeatedly can add up the error. Algorithm 7 shows our approach to get statistical guarantees: It interleaves a guided simulation phase (Lines 7-10) with a guaranteed standard bounded value iteration (called BVI phase) that uses our new functions (Lines 11-16).

The simulation phase builds the partial model by exploring states and remembering the counters. In the first iteration of the main loop, it chooses actions randomly. In all further iterations, it is guided by the bounds that the last BVI

\footnotetext{
${ }^{6}$ For $\delta_{\mathbb{T}}=0.2$, sampling the path to target once suffices to realize that $\mathrm{L}\left(\mathrm{s}_{0}, \mathrm{a}_{2}\right)>0$.
} 
phase computed. After $\mathcal{N}_{k}$ simulations (see below for a discussion of how to choose $\mathcal{N}_{k}$ ), all the gathered information is used to compute one version of the partial model with probability estimates $\widehat{\mathbb{T}}$ for a certain error tolerance $\delta_{k}$. We can continue with the assumption, that these probability estimates are correct, since it is only violated with a probability smaller than our error tolerance (see below for an explanation of the choice of $\delta_{k}$ ). So in our correct partial model, we re-initialize the lower and upper bound (Line 12), and execute a guaranteed standard BVI. If the simulation phase already gathered enough data, i.e. explored the relevant states and sampled the relevant transitions often enough, this BVI achieves a precision smaller than $\varepsilon$ in the initial state, and the algorithm terminates. Otherwise we start another simulation phase that is guided by the improved bounds.

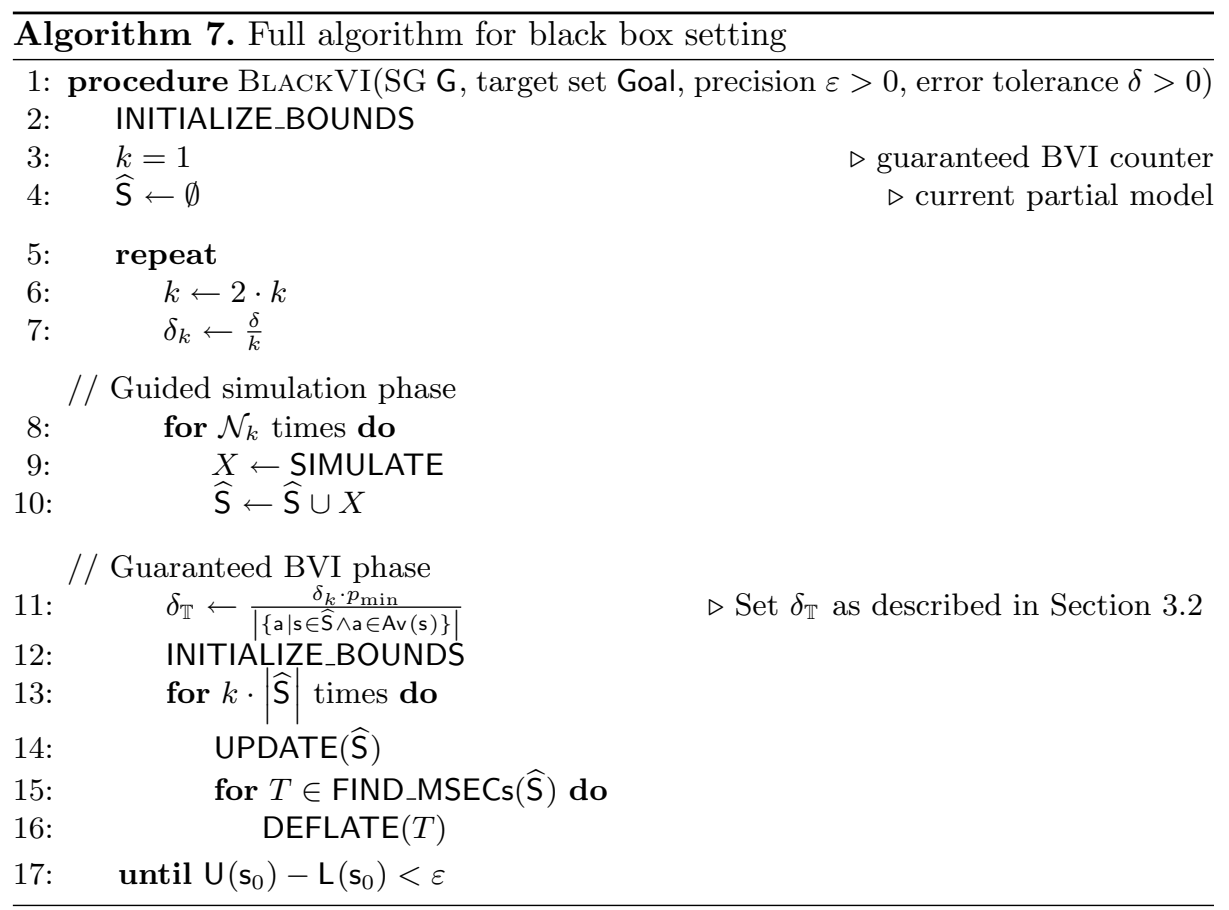

Choice of $\delta_{k}$ : For each of the full BVI phases, we construct a partial model that is correct with probability $\left(1-\delta_{k}\right)$. To ensure that the sum of these errors is not larger than the specified error tolerance $\delta$, we use the variable $k$, which is initialised to 1 and doubled in every iteration of the main loop. Hence for the $i$-th BVI, $k=2^{i}$. By setting $\delta_{k}=\frac{\delta}{k}$, we get that $\sum_{i=1}^{\infty} \delta_{k}=\sum_{i=1}^{\infty} \frac{\delta}{2^{i}}=\delta$, and hence the error of all BVI phases does not exceed the specified error tolerance. 
When to Stop Each BVI-Phase: The BVI phase might not converge if the probability estimates are not good enough. We increase the number of iterations for each BVI depending on $k$, because that way we ensure that it eventually is allowed to run long enough to converge. On the other hand, since we always run for finitely many iterations, we also ensure that, if we do not have enough information yet, BVI is eventually stopped. Other stopping criteria could return arbitrarily imprecise results [HM17]. We also multiply with $|\widehat{S}|$ to improve the chances of the early BVIs to converge, as that number of iterations ensures that every value has been propagated through the whole model at least once.

Discussion of the Choice of $\mathcal{N}_{k}$ : The number of simulations between the guaranteed BVI phases can be chosen freely; it can be a constant number every time, or any sequence of natural numbers, possibly parameterised by e.g. $k,|\widehat{S}|$, $\varepsilon$ or any of the parameters of $\mathrm{G}$. The design of particularly efficient choices or learning mechanisms that adjust them on the fly is an interesting task left for future work. We conjecture the answer depends on the given SG and "task" that the user has for the algorithm: E.g. if one just needs a quick general estimate of the behaviour of the model, a smaller choice of $\mathcal{N}_{k}$ is sensible; if on the other hand a definite precision $\varepsilon$ certainly needs to be achieved, a larger choice of $\mathcal{N}_{k}$ is required.

Theorem 1. For any choice of sequence for $\mathcal{N}_{k}$, Algorithm 7 is an anytime algorithm with the following property: When it is stopped, it returns an interval for $\mathrm{V}\left(\mathrm{s}_{0}\right)$ that is $P A C^{7}$ for the given error tolerance $\delta$ and some $\varepsilon^{\prime}$, with $0 \leq$ $\varepsilon^{\prime} \leq 1$.

Theorem 1 is the foundation of the practical usability of our algorithm. Given some time frame and some $\mathcal{N}_{k}$, it calculates an approximation for $\mathrm{V}\left(\mathrm{s}_{0}\right)$ that is probably correct. Note that the precision $\varepsilon^{\prime}$ is independent of the input parameter $\varepsilon$, and could in the worst case be always 1 . However, practically it often is good (i.e. close to 0) as seen in the results in Sect. 4. Moreover, in our modified algorithm, we can also give a convergence guarantee as in $[\mathrm{BCC}+14]$. Although mostly out of theoretical interest, in [AKW19, Appendix D.4] we design such a sequence $\mathcal{N}_{k}$, too. Since this a-priori sequence has to work in the worst case, it depends on an infeasibly large number of simulations.

Theorem 2. There exists a choice of $\mathcal{N}_{k}$, such that Algorithm 7 is PAC for any input parameters $\varepsilon, \delta$, i.e. it terminates almost surely and returns an interval for $\mathrm{V}\left(\mathrm{s}_{0}\right)$ of width smaller than $\varepsilon$ that is correct with probability at least $1-\delta$.

\footnotetext{
${ }^{7}$ Probably Approximately Correct, i.e. with probability greater than $1-\delta$, the value lies in the returned interval of width $\varepsilon^{\prime}$.
} 


\subsection{Utilizing the Additional Information of Grey Box Input}

In this section, we consider the grey box setting, i.e. for every state-action pair $(\mathrm{s}, \mathrm{a})$ we additionally know the exact number of successors $\mid$ Post $(\mathrm{s}, \mathrm{a}) \mid$. Then we can sample every state-action pair until we have seen all successors, and hence this information amounts to having qualitative information about the transitions, i.e. knowing where the transitions go, but not with which probability.

In that setting, we can improve the EC-detection and the estimated bounds in UPDATE. For EC-detection, note that the whole point of $\delta_{\mathbb{T}}$-sure EC is to check whether there are further transitions available; in grey box, we know this and need not depend on statistics. For the bounds, note that the equations for $\widehat{L}$ and $\widehat{U}$ both have two parts: The usual Bellman part and the remaining probability multiplied with the most conservative guess of the bound, i.e. 0 and 1 . If we know all successors of a state-action pair, we do not have to be as conservative; then we can use $\min _{t \in \operatorname{Post}(s, a)} \mathrm{L}(\mathrm{t})$ respectively $\max _{\mathrm{t} \in \operatorname{Post}(\mathrm{s}, \mathrm{a})} \mathrm{U}(\mathrm{t})$. Both these improvements have huge impact, as demonstrated in Sect. 4. However, of course, they also assume more knowledge about the model.

\section{Experimental Evaluation}

We implemented the approach as an extension of PRISM-Games [CFK+13a]. 11 MDPs with reachability properties were selected from the Quantitative Verification Benchmark Set $[\mathrm{HKP}+19]$. Further, 4 stochastic games benchmarks from [CKJ12,SS12, CFK+13b, CKPS11] were also selected. We ran the experiments on a 40 core Intel Xeon server running at $2.20 \mathrm{GHz}$ per core and having $252 \mathrm{~GB}$ of RAM. The tool however utilised only a single core and $1 \mathrm{~GB}$ of memory for the model checking. Each benchmark was ran 10 times with a timeout of $30 \mathrm{~min}$. We ran two versions of Algorithm 7, one with the SG as a black box, the other as a grey box (see Definition 2). We chose $\mathcal{N}_{k}=10,000$ for all iterations. The tool stopped either when a precision of $10^{-8}$ was obtained or after $30 \mathrm{~min}$. In total, 16 different model-property combinations were tried out. The results of the experiment are reported in Table 1.

In the black box setting, we obtained $\varepsilon<0.1$ on 6 of the benchmarks. 5 benchmarks were 'hard' and the algorithm did not improve the precision below 1. For 4 of them, it did not even finish the first simulation phase. If we decrease $\mathcal{N}_{k}$, the BVI phase is entered, but still no progress is made.

In the grey box setting, on 14 of 16 benchmarks, it took only 6 min to achieve $\varepsilon<0.1$. For 8 these, the exact value was found within that time. Less than $50 \%$ of the state space was explored in the case of pacman, pneuli-zuck-3, rabin-3, zeroconf and cloud_5. A precision of $\varepsilon<0.01$ was achieved on 15/16 benchmarks over a period of $30 \mathrm{~min}$. 
Table 1. Achieved precision $\varepsilon^{\prime}$ given by our algorithm in both grey and black box settings after running for a period of $30 \mathrm{~min}$ (See the paragraph below Theorem 1 for why we use $\varepsilon^{\prime}$ and not $\varepsilon$ ). The first set of the models are MDPs and the second set are SGs. '-' indicates that the algorithm did not finish the first simulation phase and hence partial BVI was not called. $m$ is the number of steps required by the DQL algorithm of $[\mathrm{BCC}+14]$ before the first update. As this number is very large, we report only $\log _{10}(m)$. For comparison, note that the age of the universe is approximately $10^{26} \mathrm{~ns}$; logarithm of number of steps doable in this time is thus in the order of 26 .

\begin{tabular}{l|l|l|l|l|l}
\hline \multirow{2}{*}{ Model } & \multirow{2}{*}{ States } & Explored \% & \multicolumn{2}{|l|}{ Precision } & \multirow{2}{*}{$\log _{10}(m)$} \\
\cline { 3 - 5 } & & Grey/Black & Grey & Black & \\
\hline consensus & 272 & $100 / 100$ & 0.00945 & 0.171 & 338 \\
\hline csma-2-2 & 1,038 & $93 / 93$ & 0.00127 & 0.2851 & 1,888 \\
\hline firewire & 83,153 & $55 /-$ & 0.0057 & 1 & 129,430 \\
\hline ij-3 & 7 & $100 / 100$ & 0 & 0.0017 & 2,675 \\
\hline ij-10 & 1,023 & $100 / 100$ & 0 & 0.5407 & 17 \\
\hline pacman & 498 & $18 / 47$ & 0.00058 & 0.0086 & 1,801 \\
\hline philosophers-3 & 956 & $56 / 21$ & 0 & 1 & 2,068 \\
\hline pnueli-zuck-3 & 2,701 & $25 / 71$ & 0 & 0.0285 & 5,844 \\
\hline rabin-3 & 27,766 & $7 / 4$ & 0 & 0.026 & 110,097 \\
\hline wlan-0 & 2,954 & $100 / 100$ & 0 & 0.8667 & 9,947 \\
\hline zeroconf & 670 & $29 / 27$ & 0.00007 & 0.0586 & 5,998 \\
\hline cdmsn & 1,240 & $100 / 98$ & 0 & 0.8588 & 3,807 \\
\hline cloud-5 & 8,842 & $49 / 20$ & 0.00031 & 0.0487 & 71,484 \\
\hline mdsm-1 & 62,245 & $69 /-$ & 0.09625 & 1 & 182,517 \\
\hline mdsm-2 & 62,245 & $72 /-$ & 0.00055 & 1 & 182,517 \\
\hline team-form-3 & 12,476 & $64 /-$ & 0 & 1 & 54,095 \\
\hline
\end{tabular}

Figure 2 shows the evolution of the lower and upper bounds in both the greyand the black box settings for 4 different models. Graphs for the other models as well as more details on the results are in [AKW19, Appendix C]. 

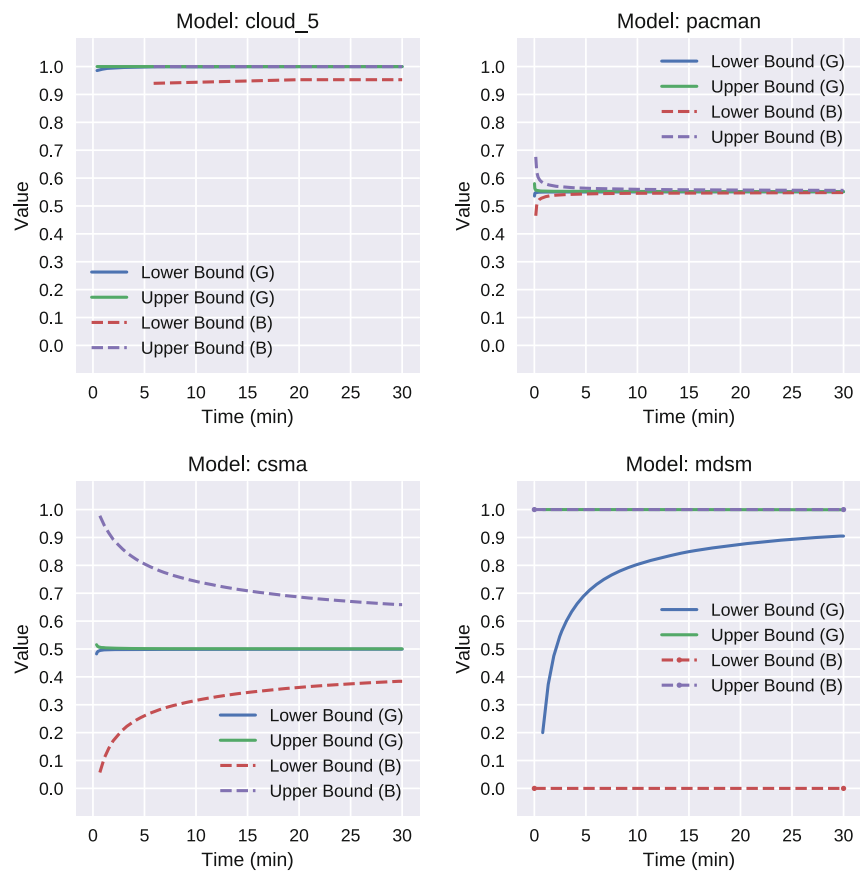

Fig. 2. Performance of our algorithm on various MDP and SG benchmarks in grey and black box settings. Solid lines denote the bounds in the grey box setting while dashed lines denote the bounds in the black box setting. The plotted bounds are obtained after each partial BVI phase, because of which they do not start at $[0,1]$ and not at time 0 . Graphs of the remaining benchmarks may be found in [AKW19, Appendix C].

\section{Conclusion}

We presented a PAC SMC algorithm for SG (and MDP) with the reachability objective. It is the first one for SG and the first practically applicable one. Nevertheless, there are several possible directions for further improvements. For instance, one can consider different sequences for lengths of the simulation phases, possibly also dependent on the behaviour observed so far. Further, the error tolerance could be distributed in a non-uniform way, allowing for fewer visits in rarely visited parts of end components. Since many systems are strongly connected, but at the same time feature some infrequent behaviour, this is the next bottleneck to be attacked. [KM19]

\section{References}

[AKW19] Ashok, P., Křetínský, J.: Maximilian Weininger. PAC statistical model checking for markov decision processes and stochastic games. Technical Report arXiv.org/abs/1905.04403 (2019) 
[BBB+10] Basu, A., Bensalem, S., Bozga, M., Caillaud, B., Delahaye, B., Legay, A.: Statistical abstraction and model-checking of large heterogeneous systems. In: Hatcliff, J., Zucca, E. (eds.) FMOODS/FORTE 2010. LNCS, vol. 6117, pp. 32-46. Springer, Heidelberg (2010). https://doi.org/10. 1007/978-3-642-13464-7_4

[BCC+14] Brázdil, T., et al.: Verification of markov decision processes using learning algorithms. In: Cassez, F., Raskin, J.-F. (eds.) ATVA 2014. LNCS, vol. 8837, pp. 98-114. Springer, Cham (2014). https://doi.org/10.1007/9783-319-11936-6_8

[BCLS13] Boyer, B., Corre, K., Legay, A., Sedwards, S.: PLASMA-lab: a flexible, distributable statistical model checking library. In: Joshi, K., Siegle, M., Stoelinga, M., DArgenio, P.R. (eds.) QEST 2013. LNCS, vol. 8054, pp. 160-164. Springer, Heidelberg (2013). https://doi.org/10.1007/9783-642-40196-1_12

[BDL+12] Bulychev, P.E., et al.: UPPAAL-SMC: statistical model checking for priced timed automata. In: QAPL (2012)

[BFHH11] Bogdoll, J., Ferrer Fioriti, L.M., Hartmanns, A., Hermanns, H.: Partial order methods for statistical model checking and simulation. In: Bruni, R., Dingel, J. (eds.) FMOODS/FORTE 2011. LNCS, vol. 6722, pp. 59-74. Springer, Heidelberg (2011). https://doi.org/10.1007/978-3-642-21461$5 \_4$

[BHH12] Bogdoll, J., Hartmanns, A., Hermanns, H.: Simulation and statistical model checking for modestly nondeterministic models. In: Schmitt, J.B. (ed.) MMB\&DFT 2012. LNCS, vol. 7201, pp. 249-252. Springer, Heidelberg (2012). https://doi.org/10.1007/978-3-642-28540-0_20

[BK08] Baier, C., Katoen, J.-P.: Principles of Model Checking. MIT Press (2008). ISBN 978-0-262-02649-9

[BT99] Brafman, R.I., Tennenholtz, M.: A near-optimal poly-time algorithm for learning a class of stochastic games. In: IJCAI, pp. 734-739 (1999)

[CFK+13a] Chen, T., Forejt, V., Kwiatkowska, M., Parker, D., Simaitis, A.: PRISMgames: a model checker for stochastic multi-player games. In: Piterman, N., Smolka, S.A. (eds.) TACAS 2013. LNCS, vol. 7795, pp. 185-191. Springer, Heidelberg (2013). https://doi.org/10.1007/978-3-642-367427_13

[CFK+13b] Chen, T., Forejt, V., Kwiatkowska, M., Parker, D., Simaitis, A.: Automatic verification of competitive stochastic systems. Formal Meth. Syst. Des. 43(1), 61-92 (2013)

[CH08] Chatterjee, K., Henzinger, T.A.: Value iteration. In: Grumberg, O., Veith, H. (eds.) 25 Years of Model Checking. LNCS, vol. 5000, pp. 107-138. Springer, Heidelberg (2008). https://doi.org/10.1007/978-3-540-69850$0 \_7$

[CH12] Chatterjee, K., Henzinger, T.A.: A survey of stochastic $\omega$-regular games. J. Comput. Syst. Sci. 78(2), 394-413 (2012)

[CKJ12] Calinescu, R., Kikuchi, S., Johnson, K.: Compositional reverification of probabilistic safety properties for large-scale complex IT systems. In: Calinescu, R., Garlan, D. (eds.) Monterey Workshop 2012. LNCS, vol. 7539, pp. 303-329. Springer, Heidelberg (2012). https://doi.org/10.1007/ 978-3-642-34059-8_16

[CKPS11] Chen, T., Kwiatkowska, M., Parker, D., Simaitis, A.: Verifying team formation protocols with probabilistic model checking. In: Leite, J., Torroni, 
P., Ågotnes, T., Boella, G., van der Torre, L. (eds.) CLIMA 2011. LNCS (LNAI), vol. 6814, pp. 190-207. Springer, Heidelberg (2011). https://doi. org/10.1007/978-3-642-22359-4_14

[Con92] Condon, A.: The complexity of stochastic games. Inf. Comput. 96(2), 203-224 (1992)

[CZ11] Clarke, E.M., Zuliani, P.: Statistical model checking for cyber-physical systems. In: ATVA, pp. 1-12 (2011)

[DDL+12] David, A., et al.: Statistical model checking for stochastic hybrid systems. In: HSB, pp. 122-136 (2012)

[DDL+13] David, A., Du, D., Guldstrand Larsen, K., Legay, A., Mikučionis, M.: Optimizing control strategy using statistical model checking. In: Brat, G., Rungta, N., Venet, A. (eds.) NFM 2013. LNCS, vol. 7871, pp. 352-367. Springer, Heidelberg (2013). https://doi.org/10.1007/978-3-642-380884_24

[DHKP16] Daca, P., Henzinger, T.A., Křetínský, J., Petrov, T.: Faster statistical model checking for unbounded temporal properties. In: Chechik, M., Raskin, J.-F. (eds.) TACAS 2016. LNCS, vol. 9636, pp. 112-129. Springer, Heidelberg (2016). https://doi.org/10.1007/978-3-662-496749_7

[DHS18] D'Argenio, P.R., Hartmanns, A., Sedwards, S.: Lightweight statistical model checking in nondeterministic continuous time. In: Margaria, T., Steffen, B. (eds.) ISoLA 2018. LNCS, vol. 11245, pp. 336-353. Springer, Cham (2018). https://doi.org/10.1007/978-3-030-03421-4_22

[DLL+11a] David, A., et al.: Statistical model checking for networks of priced timed automata. In: Fahrenberg, U., Tripakis, S. (eds.) FORMATS 2011. LNCS, vol. 6919, pp. 80-96. Springer, Heidelberg (2011). https://doi.org/10. 1007/978-3-642-24310-3_7

[DLL+11b] David, A., Larsen, K.G., Legay, A., Mikučionis, M., Wang, Z.: Time for statistical model checking of real-time systems. In: Gopalakrishnan, G., Qadeer, S. (eds.) CAV 2011. LNCS, vol. 6806, pp. 349-355. Springer, Heidelberg (2011). https://doi.org/10.1007/978-3-642-22110-1_27

[DLST15] D'Argenio, P., Legay, A., Sedwards, S., Traonouez, L.-M.: Smart sampling for lightweight verification of markov decision processes. STTT 17(4), 469-484 (2015)

[EGF12] Ellen, C., Gerwinn, S., Fränzle, M.: Confidence bounds for statistical model checking of probabilistic hybrid systems. In: Jurdziński, M., Ničković, D. (eds.) FORMATS 2012. LNCS, vol. 7595, pp. 123-138. Springer, Heidelberg (2012). https://doi.org/10.1007/978-3-642-33365$1 \_10$

[FT14] Fu, J., Topcu, U.: Probably approximately correct MDP learning and control with temporal logic constraints. In: Robotics: Science and Systems (2014)

[HAK18] Hasanbeig, M., Abate, A., Kroening, D.: Logically-correct reinforcement learning. CoRR, 1801.08099 (2018)

[HAK19] Hasanbeig, M., Abate, A., Kroening, D.: Certified reinforcement learning with logic guidance. CoRR, abs/1902.00778 (2019)

[HJB+10] He, R., Jennings, P., Basu, S., Ghosh, A.P., Wu, H.: A bounded statistical approach for model checking of unbounded until properties. In: ASE, pp. $225-234$ (2010) 
[HKP+19] Hartmanns, A., Klauck, M., Parker, D., Quatmann, T., Ruijters, E.: The quantitative verification benchmark set. In: TACAS 2019 (2019, to appear)

[HLMP04] Hérault, T., Lassaigne, R., Magniette, F., Peyronnet, S.: Approximate probabilistic model checking. In: Steffen, B., Levi, G. (eds.) VMCAI 2004. LNCS, vol. 2937, pp. 73-84. Springer, Heidelberg (2004). https://doi.org/ 10.1007/978-3-540-24622-0_8

[HM17] Haddad, S., Monmege, B.: Interval iteration algorithm for MDPs and IMDPs. Theor. Comput. Sci. (2017)

[HMZ+12] Henriques, D., Martins, J., Zuliani, P., Platzer, A., Clarke, E.M.: Statistical model checking for Markov decision processes. In: QEST, pp. 84-93 (2012)

[HPS+19] Hahn, E.M., Perez, M., Schewe, S., Somenzi, F., Trivedi, A., Wojtczak, D.: Omega-regular objectives in model-free reinforcement learning. In: Vojnar, T., Zhang, L. (eds.) TACAS 2019. LNCS, vol. 11427, pp. 395412. Springer, Cham (2019). https://doi.org/10.1007/978-3-030-17462$0 \_27$

[JCL+09] Jha, S.K., Clarke, E.M., Langmead, C.J., Legay, A., Platzer, A., Zuliani, P.: A bayesian approach to model checking biological systems. In: Degano, P., Gorrieri, R. (eds.) CMSB 2009. LNCS, vol. 5688, pp. 218-234. Springer, Heidelberg (2009). https://doi.org/10.1007/978-3-642-038457_15

[JLS12] Jegourel, C., Legay, A., Sedwards, S.: A platform for high performance statistical model checking - PLASMA. In: Flanagan, C., König, B. (eds.) TACAS 2012. LNCS, vol. 7214, pp. 498-503. Springer, Heidelberg (2012). https://doi.org/10.1007/978-3-642-28756-5_37

[KKKW18] Kelmendi, E., Krämer, J., Křetínský, J., Weininger, M.: Value iteration for simple stochastic games: stopping criterion and learning algorithm. In: Chockler, H., Weissenbacher, G. (eds.) CAV 2018. LNCS, vol. 10981, pp. 623-642. Springer, Cham (2018). https://doi.org/10.1007/978-3-31996145-3_36

[KM19] Křetínský, J., Meggendorfer, T.: Of cores: a partial-exploration framework for Markov decision processes. Submitted 2019

[KNP11] Kwiatkowska, M., Norman, G., Parker, D.: PRISM 4.0: verification of probabilistic real-time systems. In: Gopalakrishnan, G., Qadeer, S. (eds.) CAV 2011. LNCS, vol. 6806, pp. 585-591. Springer, Heidelberg (2011). https://doi.org/10.1007/978-3-642-22110-1_47

[Lar12] Larsen, K.G.: Statistical model checking, refinement checking, optimization,. for stochastic hybrid systems. In: Jurdziński, M., Ničković, D. (eds.) FORMATS 2012. LNCS, vol. 7595, pp. 7-10. Springer, Heidelberg (2012). https://doi.org/10.1007/978-3-642-33365-1_2

[Lar13] Guldstrand Larsen, K.: Priced timed automata and statistical model checking. In: Johnsen, E.B., Petre, L. (eds.) IFM 2013. LNCS, vol. 7940, pp. 154-161. Springer, Heidelberg (2013). https://doi.org/10.1007/9783-642-38613-8_11

[Lit94] Littman, M.L.: Markov games as a framework for multi-agent reinforcement learning. In: ICML, pp. 157-163 (1994)

[LN81] Lakshmivarahan, S., Narendra, K.S.: Learning algorithms for two-person zero-sum stochastic games with incomplete information. Math. Oper. Res. 6(3), 379-386 (1981) 
[LP08] Lassaigne, R., Peyronnet, S.: Probabilistic verification and approximation. Ann. Pure Appl. Logic 152(1-3), 122-131 (2008)

[LP12] Lassaigne, R., Peyronnet, S.: Approximate planning and verification for large Markov decision processes. In: SAC, pp. 1314-1319, (2012)

[LST14] Legay, A., Sedwards, S., Traonouez, L.-M.: Scalable verification of markov decision processes. In: Canal, C., Idani, A. (eds.) SEFM 2014. LNCS, vol. 8938, pp. 350-362. Springer, Cham (2015). https://doi.org/10.1007/9783-319-15201-1_23

[Mar75] Martin, D.A.: Borel determinacy. Ann. Math. 102(2), 363-371 (1975)

[MLG05] Mcmahan, H.B., Likhachev, M., Gordon, G.J.: Bounded real-time dynamic programming: RTDP with monotone upper bounds and performance guarantees. In: In ICML 2005, pp. 569-576 (2005)

[Nor98] Norris, J.R.: Markov Chains. Cambridge University Press, Cambridge (1998)

[PGL+13] Palaniappan, S.K., Gyori, B.M., Liu, B., Hsu, D., Thiagarajan, P.S.: Statistical model checking based calibration and analysis of bio-pathway models. In: Gupta, A., Henzinger, T.A. (eds.) CMSB 2013. LNCS, vol. 8130, pp. 120-134. Springer, Heidelberg (2013). https://doi.org/10.1007/ 978-3-642-40708-6_10

[Put14] Puterman, M.L.: Markov Decision Processes: Discrete Stochastic Dynamic Programming. Wiley, Hoboken (2014)

[RF91] Raghavan, T.E.S., Filar, J.A.: Algorithms for stochastic games - a survey. Z. Oper. Res. 35(6), 437-472 (1991)

[RP09] El Rabih, D., Pekergin, N.: Statistical model checking using perfect simulation. In: Liu, Z., Ravn, A.P. (eds.) ATVA 2009. LNCS, vol. 5799, pp. 120-134. Springer, Heidelberg (2009). https://doi.org/10.1007/9783-642-04761-9_11

[SB98] Sutton, R., Barto, A.: Reinforcement Learning: An Introduction. MIT Press, Cambridge (1998)

[SKC+14] Sadigh, D., Kim, E.S., Coogan, S., Sastry, S.S.S., Sanjit, A.: A learning based approach to control synthesis of markov decision processes for linear temporal logic specifications. In: CDC, pp. 1091-1096 (2014)

[SLW+06] Strehl, A.L., Li, L., Wiewiora, E., Langford, J., Littman, M.L.: PAC model-free reinforcement learning. In: ICML, pp. 881-888 (2006)

[SS12] Saffre, F., Simaitis, A.: Host selection through collective decision. ACM Trans. Auton. Adapt. Syst. 7(1), 4:1-4:16 (2012)

[SVA04] Sen, K., Viswanathan, M., Agha, G.: Statistical model checking of blackbox probabilistic systems. In: Alur, R., Peled, D.A. (eds.) CAV 2004. LNCS, vol. 3114, pp. 202-215. Springer, Heidelberg (2004). https://doi. org $/ 10.1007 / 978-3-540-27813-9 \_16$

[SVA05] Sen, K., Viswanathan, M., Agha, G.: On statistical model checking of stochastic systems. In: Etessami, K., Rajamani, S.K. (eds.) CAV 2005. LNCS, vol. 3576, pp. 266-280. Springer, Heidelberg (2005). https://doi. org/10.1007/11513988_26

[WT16] Wen, M., Topcu, U.: Probably approximately correct learning in stochastic games with temporal logic specifications. In: IJCAI, pp. 3630-3636 (2016)

[YCZ10] Younes, H.L.S., Clarke, E.M., Zuliani, P.: Statistical verification of probabilistic properties with unbounded until. In: Davies, J., Silva, L., Simao, A. (eds.) SBMF 2010. LNCS, vol. 6527, pp. 144-160. Springer, Heidelberg (2011). https://doi.org/10.1007/978-3-642-19829-8_10 
[YKNP06] Younes, H.L.S., Kwiatkowska, M.Z., Norman, G., Parker, D.: Numerical vs. statistical probabilistic model checking. STTT 8(3), 216-228 (2006)

[YS02a] Younes, H.L.S., Simmons, R.G.: Probabilistic verification of discrete event systems using acceptance sampling. In: Brinksma, E., Larsen, K.G. (eds.) CAV 2002. LNCS, vol. 2404, pp. 223-235. Springer, Heidelberg (2002). https://doi.org/10.1007/3-540-45657-0_17

[ZPC10] Zuliani, P., Platzer, A., Clarke, E.M.: Bayesian statistical model checking with application to simulink/stateflow verification. In: HSCC, pp. 243$252(2010)$

Open Access This chapter is licensed under the terms of the Creative Commons Attribution 4.0 International License (http://creativecommons.org/licenses/by/4.0/), which permits use, sharing, adaptation, distribution and reproduction in any medium or format, as long as you give appropriate credit to the original author(s) and the source, provide a link to the Creative Commons license and indicate if changes were made.

The images or other third party material in this chapter are included in the chapter's Creative Commons license, unless indicated otherwise in a credit line to the material. If material is not included in the chapter's Creative Commons license and your intended use is not permitted by statutory regulation or exceeds the permitted use, you will need to obtain permission directly from the copyright holder. 